\title{
AUTHOR INDEX VOLUME 12 (2003)
}

Alatas H, Iskandar AA, Tjia MO \& Valkering TP, Analytic Study of Stationary Solitons in Deep Nonlinear Bragg Grating

Andonowati \& van Groesen E, Optical Pulse Deformation in Second Order Nonlinear Media

12 (2003) 221

Assanto G, Peccianti M, Brzdąkiewicz KA, de Luca A \& Umeton C, Nonlinear Wave Propagation and Spatial Solitons in Nematic

Liquid Crystals

Assanto G, see Peccianti $M$

Bai F, see Qin $A$

Bai Y, see Zhang $G$

Barberi R, see Blinov LM

Barhai PK, see Konar $S$

Biswas A, Dispersion-Managed Solitons in Optical Couplers

Biswas A, Gabitov-Turitsyn Equation for Solitons in Optical Fibers

Blinov LM, Barberi R, Ciuchi F, De Santo MP, Cipparone G, Mazzulla A, Palto SP \& Yudin SG, Unpolar and Polar Holographic Grating Recording by Circularly Polarized Light on Photoanisotropic Azobenzene Langmuir-Blodgett Films

Bosshard C, see Stegeman GI

Brenier A, Optimization of GdCOB:Nd and YCOB:Nd Crystals for Self-Frequency Doubling Out of the Principal Planes

Brzda̧kiewicz KA, see Assanto $G$

Carrasco S, see Stegeman GI

Cattaneo S, Siltanen M \& Kauranen M, New Polarization Techniques for Precise Characterization of Second-Order Thin Films

Chan KC, see Mahmood Mat Yunus W

Chen $\mathrm{K}$, see Khoo IC

Chen W, see Zhang $G$

Cipparone G, see Blinov LM

Ciuchi F, see Blinov LM

Clays K, Molecular Nonlinear Optics: From Para-Nitroaniline to Electrochemical Switching of the Hyperpolarizability

12 (2003) 45

12 (2003) 17

Collings N, see Ruzak $O$

Conti C, see Peccianti $M$

Crossland WA, see Ruzak $O$

Davey AB, see Ruzak $O$

de Luca A, see Assanto $G$

De Luca A, see Peccianti $M$

De Santo MP, see Blinov LM

Debrus S, see Marchewka $M K$

Diaz A, see Khoo IC

Ding J, see Khoo IC

Ding YJ \& Shi W, Widely-Tunable, Monochromatic, and High-Power Terahertz Sources and Their Applications

12 (2003) 557

Driessen A, see Tan FS

Dyadyusha A, see Kaczmarek $M$ 
Eger D, see Stegeman GI

Elim HI, Ji W, Meng GC, Ouyang J-Y \& Goh SH, Nonlinear Optics and Optical Limiting Properties of Multifunctional Fullerenol/Polymer Composite

$12(2003) 175$

Freydank AC, see Samoc A

Gao S, Yang C \& Jin G, Comparison of Interchannel Four-Wave Mixing with Continuous Waves, Quasi-Continuous Waves and Ultrashort Pulses

Genov DA, Sarychev AK \& Shalaev VM, Metal-Dielectric Composite Filters with Controlled Spectral Windows of Transparency

George DS, see Kurian A

Goh SH, see Elim HI

Gunter P, see Stegeman GI

Guo S-L, Wang H-T, Ming NB, Zhang JY \& Guo ZJ, Investigation of Near Two-Photon Resonance Optical Nonlinearities in $\mathrm{Ni}(\mathrm{Ac})_{2}[\mathrm{~N}$-(8-Quinolyl) Pyridine-2-Carboxamide $] \cdot 4 \mathrm{H}_{2} \mathrm{O}$ by the Z-Scan Technique

Guo S-L, Xu L, Yan J, Wang H-T, You X-Z \& Ming NB, An Accurate Method for Extracting Nonlinear Refraction by Z-Scan Technique in the Presence of Nonlinear Absorption

Guo ZJ, see Guo $S-L$

Hammer M, see Suryanto A

Hanamura E, Kawabe Y, Takashima H, Sato T \& Tomita A, Optical Properties of Transition-Metal Doped Spinels

He J, see $M a G$

Herman, see Taunaumang $H$

Hidayat R, see Taunaumang $H$

Hoekstra HJWM, see Tan FS

Hoekstra HJWM, see Uranus HP

Hollink A, see Tan FS

$\mathrm{Hu}$ Y-H, see Zhou J-Y

Ikeda T, see Okano $K$

Iskandar AA, see Alatas $H$

Iskandar AA, see Prawiharjo $J$

Jankovic L, see Stegeman GI

Ji W, see Elim HI

Jin G, see Gao $S$

Joshi MP, see Tiwari SK

Kaczmarek M \& Dyadyusha A, Structured, Photosensitive PVK and PVCN Polymer Layers for Control of Liquid Crystal Alignment

Katz M, see Stegeman GI

Kauranen M, see Cattaneo $S$

Kawabe Y, see Hanamura E

Kelderman H, see Tan FS

Keller O, Optical Near-Field Interaction on the Basis of Photon Wave Mechanics

Khoo IC, Diaz A, Ding J, Chen K \& Zhang Y, Collective and Individual Molecular Nonlinear Photonics of Liquid Crystals

Khoo IC, see Ruzak $O$

Kim H, see Stegeman GI

Klunder DJW, see Tan FS 
Konar S, Barhai PK \& Medhekar S, Displacement and Deflection of Optical Beams by Nonlinear Planar Waveguide

Kumar GA, Nonlinear Optical Response and Reverse Saturable Absorption of Rare Earth Phthalocyanine in DMF Solution

Kuriakose VC, see Vinoj $M N$

12 (2003) 367

Kurian A, Lee ST, Unnikrishnan KP, George DS, Nampoori VPN \& Vallabhan CPG, Studies on Two-Photon Absorption of Aniline Using Thermal Lens Effect

Lee ST, see Kurian $A$

Li L, see Xiao $Y$

Li Z, see Xiao $Y$

Lin HK \& Lue J-T, Dynamic Oscillation of Optical Transmittance of Nematic Liquid Crystals During the Turn-on and Turn-off of Applying Field and Temperature Variation

12 (2003) 323

Lucas NT, see Samoc A

Lue J-T, see Lin $H K$

Luther-Davies B, see Samoc A

Ma G, He J, Tang S-H, Sun W-X \& Shen Z-X, Size-Dependence of Nonlinearity in Metal: Dielectric Composite System Induced by Local Field Enhancement

Mahmood Mat Yunus W, Chan KC \& Md. Zin Wan Yunus W,Study on Photobleaching of Methylene Blue Doped in PMMA, PVA and Gelatin Using Photoacoustic Technique

Marchewka MK, Ratajczak H \& Debrus S, Infrared Spectrum and Nonlinear Optical Properties of p-Nitroaniline-L-Tartaric Acid (2:1) Molecular Complex

Mazzulla A, see Blinov LM

Md. Zin Wan Yunus W, see Mahmood Mat Yunus W

Medhekar S, see Konar $S$

Mehendale SC, see Tiwari SK

Meng GC, see Elim HI

Ming NB, see Guo $S-L$

Ming NB, see Guo $S$-L

Misra SCK, see Sharma SK

Misra SCK, see Sharma $S K$

Nampoori VPN, see Kurian A

Nath S, see Tiwari $S K$

Neff CW, see Summers CJ

Okano K, Shishido A, Tsutsumi O, Shiono T \& Ikeda T, Highly Efficient Holographic Materials Based on Polymer Liquid Crystals Ouyang J-Y, see Elim HI

Palto SP, see Blinov LM

Park W, see Summers CJ

Peccianti M, see Assanto $G$

Peccianti M, Conti C, Assanto G, De Luca A \& Umeton C, Nonlocal Optical Propagation in Nonlinear Nematic Liquid Crystals

Polyakov S, see Stegeman GI

Porsezian K \& Senthilnathan K, Analysis of Potential Energy in Birefringent Fiber Through Undamped Duffing Oscillator Equation

12 (2003) 539

12 (2003) 525

$12(2003) 7$ 
Prawiharjo J, Iskandar AA, Tjia MO \& van Groesen E, Second Order Approximation for Band Gap Characterization of One-Dimensional Dielectric Omnidirectional Reflector

Qin A, Bai F \& Ye C, Enhancement of Effective Second-Order Nonlinearity in Poled Polymers by Introducing the Chromophores with Low Ground State Dipole Moment

Ratajczak H, see Marchewka $M K$

Ruzak O, Collings N, Crossland WA, Wilkinson TD, Davey AB and Khoo IC, Dynamic Holographic Gratings in Methyl Red-Doped Nematic Liquid Crystals

Samoc A, Samoc M, Luther-Davies B, Freydank AC \& Lucas NT, Investigations of Nonlinear Optical Chromophores with Femtosecond Degenerate Four-Wave Mixing in Solutions

Samoc M, see Samoc A

Sarychev AK, see Genov DA

Sato T, see Hanamura $E$

Seema AU, see Vinoj $M N$

Sengo G, see Tan FS

Senthilnathan K, see Porsezian $K$

Shalaev VM, see Genov DA

Sharma SK, Misra SCK \& Tripathi KN, Nonlinear Optical Devices: Properties of Polyaniline Based Conducting Polymers

Sharma SK, Misra SCK \& Tripathi KN, Polycyanurate Thin Film Optical Waveguide for Integrated Optics Applications

Shen Z-X, see $M a G$

Shi W, see Ding $Y J$

Shiono T, see Okano $K$

Shishido A, see Okano $K$

Siltanen M, see Cattaneo $S$

Sopaheluwakan A, see van Groesen E

Stegeman GI, Jankovic L, Kim H, Polyakov S, Carrasco S, Torner L, Bosshard C, Gunter P, Katz M \& Eger D, Generation of, and Interactions Between, Quadratic Spatial Solitons in Non-CriticallyPhase-Matched Crystals

12 (2003) 447

Summers CJ, Neff CW \& Park W, Active Photonic Crystal Nano-Architectures

$12(2003) 39$

$12(2003) 1$

Sun W-X, see $M a G$

Suryanto A, van Groesen E \& Hammer M, Finite Element Analysis of Optical Bistability in One-Dimensional Nonlinear Photonic Band Gap Structures with a Defect

12 (2003) 187

Takashima H, see Hanamura E

Tan FS, Klunder DJW, Sengo G, Kelderman H, Hollink A, Hoekstra HJWM \& Driessen A, Characterization of Vertically Waveguide Coupled Microring Resonators by Means of Quantitative Image Analysis

12 (2003) 205

Tang G, see Zhang G

Tang S-H, see $M a G$

Taunaumang H, Hidayat R, Herman \& Tjia MO, Effects of Substrate Temperature and External Poling Field on Molecular Orientation and Aggregation in Vacuum Deposited Photo Responsive DR1 Films 
Tiwari SK, Joshi MP, Nath S \& Mehendale SC, Salt-Induced Aggregation and Enhanced Optical Limiting in Carbon-Black Suspensions

$12(2003) 335$

Tjia MO, see Alatas $H$

Tjia MO, see Prawiharjo $J$

Tjia MO, see Taunaumang $H$

Tomita A, see Hanamura E

Torner L, see Stegeman GI

Tripathi KN, see Sharma SK

Tripathi KN, see Sharma SK

Tsutsumi O, see Okano $K$

Umeton C, see Assanto $G$

Umeton $\mathrm{C}$, see Peccianti $M$

Unnikrishnan KP, see Kurian A

Uranus HP, Hoekstra HJWM \& van Groesen E, Fourth-Order

Variational Mode Solving for Anisotropic Planar Structures

Valkering TP, see Alatas $H$

Vallabhan CPG, see Kurian A

van Groesen E \& Sopaheluwakan A, Extremal Characterization of

Band Gaps in Nonlinear Gratings

van Groesen E, see Andonowati

van Groesen E, see Prawiharjo $J$

van Groesen E, see Suryanto A

van Groesen E, see Uranus HP

Vinoj MN, Seema AU \& Kuriakose VC, Optimum Dispersion Profile and Pedestal-Free Soliton Pulse Compression in Dispersion-Decreasing Fiber

Wang H-T, see Guo $S$ - $L$

Wang H-T, see Guo $S-L$

Wang L, see Zhang $G$

Wang W-J, see Zhou J-Y

Wilkinson TD, see Ruzak $O$

Xiao Y, Xu Z, Li L, Li Z \& Zhou G, Soliton Propagation in Nonuniform Optical Fibers

Xiong F, see Zhang $G$

$\mathrm{Xu} \mathrm{L}$, see Guo $S-L$

$\mathrm{Xu} \mathrm{Z}$, see Xiao $Y$

Yan J, see Guo $S$ - $L$

Yang C, see Gao $S$

Ye C, see Qin A

You X-Z, see Guo $S$ - $L$

Yudin SG, see Blinov LM

Zhang B, see Zhang $G$

Zhang G, Xiong F, Zhang B, Tang G, Chen W, Wang L \& Bai Y, Effect of Solvent on Nonlinear Refractive Index of 2-(2'-Hydroxyphenyl) Benzoxazole

$12(2003) 135$

$12(2003) 291$

Zhang JY, see Guo S-L

Zhang $\mathrm{Y}$, see Khoo IC

Zhou G, see Xiao $Y$

Zhou J-Y, Wang W-J \& Hu Y-H, Study of Self-Pumped Phase Conjugations and Their Suppressions at the Common Input of Two Lasers 